\title{
Association Between Neck Circumference and Microalbuminuria in Community Residents
}

\author{
Chaohui Jian* \\ Yiting $\mathrm{Xu}^{*}$ \\ Yun Shen \\ Yufei Wang \\ Xiaojing Ma \\ Yuqian Bao
}

Department of Endocrinology and Metabolism, Shanghai Jiao Tong University Affiliated Sixth People's Hospital; Shanghai Clinical Center for Diabetes; Shanghai Key Clinical Center for Metabolic Disease; Shanghai Diabetes Institute; Shanghai Key Laboratory of Diabetes Mellitus, Shanghai, 200233, People's Republic of China

*These authors contributed equally to this work
Correspondence: Xiaojing Ma; Yuqian Bao Department of Endocrinology and Metabolism, Shanghai Jiao Tong University Affiliated Sixth People's Hospital, Shanghai Diabetes Institute, 600 Yishan Road, Shanghai, 200233, People's Republic of China

Tel +86-2I-64369l8I

Fax +86-2I-6436803I

Email maxiaojing@sjtu.edu.cn;

yqbao@sjtu.edu.cn
Objective: Neck circumference (NC) represents the subcutaneous fat deposition in the neck and is an effective indicator for evaluating metabolic disorders, such as metabolic syndrome, subclinical atherosclerosis, and non-alcoholic fatty liver disease. Microalbuminuria (MAU) is regarded as a potential sign of systemic endothelial dysfunction and microvascular abnormalities. The aim of this study was to elucidate the association of NC with urine albumin-to-creatinine ratio (UACR) and MAU.

Methods: A total of 1882 Shanghai community residents were enrolled (816 men and 1066 women), with age ranging from 40 to 80 years. Anthropometric parameters, including NC, and biochemical indices were measured. MAU was determined if $30 \mathrm{mg} / \mathrm{g} \leq \mathrm{UACR}<$ $300 \mathrm{mg} / \mathrm{g}$. An elevated $\mathrm{NC}$ was defined as $\mathrm{NC} \geq 38.5 \mathrm{~cm}$ for men and $\mathrm{NC} \geq 34.5 \mathrm{~cm}$ for women.

Results: Individuals with an elevated NC had significantly higher prevalence of MAU and UACR values than those with normal NC in both men and women (all $P<0.05$ ). The logistic regression analysis showed that there were significant and positive associations between elevated $\mathrm{NC}$ and the increasing risk of MAU after adjusting for lipid profile and glycemic indices $(P=0.007$ for men and $P=0.009$ for women). After further adjusting for blood pressure, elevated NC caused an $69.3 \%$ additional risk of MAU in men $(P=0.037)$ and the positive correlation in women disappeared $(P=0.131)$.

Conclusion: There was an independent and positive association between elevated $\mathrm{NC}$ and the risk of MAU in men in the Chinese community population.

Chinese Clinical Trial Registry (Www.chictr.org.cn) Registration Number: ChiCTR1900024011.

Keywords: neck circumference, microalbuminuria, urine albumin-to-creatinine ratio

\section{Introduction}

Neck circumference (NC), the girth measured just below the thyroid cartilage protrusion, represents the subcutaneous fat deposition in the neck, which is an anthropological parameter for determining obesity (especially upper-body obesity). $\mathrm{NC}$ has gradually attracted considerable attention from researchers and clinicians owing to its strengths, including simple measurement procedure, explicit anatomic landmark, and low variability. A growing body of evidence suggested that, similar to waist circumference (WC), NC also acted as a powerful tool in identifying metabolic syndrome, ${ }^{1,2}$ assessing subclinical atherosclerosis, ${ }^{3}$ screening for nonalcoholic fatty liver disease, ${ }^{4}$ and predicting cardiovascular diseases effectively. ${ }^{5,6}$

Microalbuminuria (MAU), which is usually measured using urine albumin-tocreatinine ratio (UACR), is seen at several instances, such as abnormal 
hemodynamics, metabolic disorders, or inflammation, ${ }^{7}$ and often indicates systemic endothelial dysfunction and microvascular abnormalities. ${ }^{8}$ The occurrence of MAU in patients with hypertension or diabetes is related to the hemodynamic adaptations of glomerular hypertension and hyperfiltration in remnant nephrons owing to glomerular permeability dysfunction. ${ }^{9,10}$ In addition, a study has shown that MAU increases the risks of cardiovascular diseases and mortality independently in the general population. ${ }^{11} \mathrm{MAU}$ is reversible in the early stage of microvascular changes, and it can regress after blood pressure, blood glucose level, obesity, or inflammation are brought under control. ${ }^{7}$ Therefore, early detection of MAU and timely control of its cause are essential to prevent the exacerbation in microvascular abnormalities to irreversible atherosclerosis and the occurrence of renal damage.

We obtained the optimal NC cutoff points for metabolic syndrome in a previous study through assessing central obesity using the precise standard-visceral fat area, which was measured utilizing magnetic resonance imaging, ${ }^{1}$ and verified its efficiency in determining subclinical atherosclerosis. ${ }^{3}$ A study has demonstrated that $\mathrm{NC}$, an index closely linked to cardiovascular diseases, was independently associated with the risk of decreased estimated glomerular filtration rate (eGFR) in the Chinese general population. ${ }^{12}$ However, whether $\mathrm{NC}$ can also be used to assess the risk of MAU in the Chinese population is yet unknown. Therefore, this study aimed to clarify the association of NC with UACR and MAU in community residents.

\section{Materials and Methods Study Population}

This study recruited Shanghai community residents between 2015 and 2016. All participants completed standardized questionnaires, which included medical history, medications, and personal habits. The exclusion criteria of this study included neck deformity or surgical history, goiter, abnormal thyroid function, and history of cardiovascular diseases, severe hepatic dysfunction, macroalbuminuria (UACR $\geq 300 \mathrm{mg} / \mathrm{g}$ ), malignant tumors, infection status, and hormone-based treatments. Finally, a total of 1882 individuals (816 men and 1066 women) with complete data were included in the study. This study was approved by the Ethics Committee of Shanghai Jiao Tong University Affiliated Sixth People's Hospital (Approval number: 2019-067) and all participants signed the informed consent.

\section{Anthropometric and Biochemical Measurements}

The measurements of anthropometric parameters, including height, weight, $\mathrm{WC}$, and $\mathrm{NC}$ were conducted in accordance with the standardized methods described in a previous study. ${ }^{1}$ Body mass index $(\mathrm{BMI})=$ weight $(\mathrm{kg}) / \mathrm{height}^{2}$ $\left(\mathrm{m}^{2}\right)$. Systolic blood pressure (SBP) and diastolic blood pressure (DBP) were measured after a minimum 10-min rest and the average of three measurements taken at 3-min intervals was recorded.

After overnight fasting for $10 \mathrm{~h}$, venous blood samples were collected, and blood samples were taken again after 2 $\mathrm{h}$ by performing a 75 -g oral glucose tolerance test or 100 -g steamed bread meal test (for valid diabetic patients). Fasting plasma glucose (FPG), 2-h plasma glucose (2hPG), glycated hemoglobin $\mathrm{A}_{1 \mathrm{c}}\left(\mathrm{HbA}_{1 \mathrm{c}}\right)$, fasting insulin, total cholesterol (TC), triglyceride (TG), high-density lipoprotein cholesterol (HDL-c), low-density lipoprotein cholesterol (LDL-c), and C-reactive protein (CRP) were measured based on standard methods as previously described. ${ }^{1}$ The homeostasis model assessment-insulin resistance index (HOMA-IR) $=$ FPG $(\mathrm{mmol} / \mathrm{L}) \times$ fasting insulin $(\mathrm{mU} / \mathrm{L}) / 22.5$. The sarcosine oxidase-PAP method was used to determine the serum and urine creatinine levels on a 7600-120 Hitachi automatic analyzer (Hitachi, Tokyo, Japan). Urine albumin content was determined using immunonephelometry (BN II System; Siemens, Marburg, Germany). UACR was the ratio of urinary albumin to urine creatinine. The eGFR was calculated by the formula proposed by the Chronic Kidney Disease Epidemiology Collaboration: $\mathrm{eGFR}=141 \times \min (\mathrm{Scr} / \kappa, 1)^{\alpha} \times \max (\mathrm{Scr} / \kappa$, $1)^{-1.209} \times 0.993^{\text {Age }} \times 1.018$ [if female] ${ }_{-} 1.159$ [if black], where Scr is serum creatinine, $\kappa$ is 0.7 for females and 0.9 for males, $\alpha$ is -0.329 for females and -0.411 for males, min indicates the minimum of $\mathrm{Scr} / \kappa$ or 1 , and max indicates the maximum of $\mathrm{Scr} / \kappa$ or $1 .^{13}$

\section{Diagnosis Criteria}

1) Diabetes was diagnosed according to the criteria set by the 2010 American Diabetes Association, FPG $\geq 7.0$ $\mathrm{mmol} / \mathrm{L}$ and $/$ or $2 \mathrm{hPG} \geq 11.1 \mathrm{mmol} / \mathrm{L}$ and/or $\mathrm{HbA}_{1 \mathrm{c}} \geq$ $6.5 \%{ }^{14}$ 2) Based on the 2020 International Society of Hypertension global hypertension practice guidelines, $\mathrm{SBP} \geq 140 \mathrm{mmHg}$ and/or DBP $\geq 90 \mathrm{mmHg}$ or currently receiving antihypertensive therapy were diagnosed as patients with hypertension. ${ }^{15}$ 3) Elevated $\mathrm{NC}$ was defined as $\mathrm{NC} \geq 38.5 \mathrm{~cm}$ for men and $\mathrm{NC} \geq 34.5 \mathrm{~cm}$ for women in the light of our previous results. ${ }^{1}$ 4) MAU was diagnosed 
if $30 \mathrm{mg} / \mathrm{g} \leq \mathrm{UACR}<300 \mathrm{mg} / \mathrm{g} .{ }^{16}$ 5) According to the latest 2020 China Guideline, metabolic syndrome was diagnosed when any of the three following components were present: central obesity (WC $\geq 90 \mathrm{~cm}$ for men and $\geq$ $85 \mathrm{~cm}$ for women); hyperglycemia: $\mathrm{FPG} \geq 6.10 \mathrm{mmol} / \mathrm{L}$ and (or) $2 \mathrm{hPG} \geq 7.80 \mathrm{mmol} / \mathrm{L}$, and (or) previously diagnosed diabetes; hypertension: $\mathrm{SBP} \geq 130 \mathrm{mmHg}$, and (or) $\mathrm{DBP} \geq 85 \mathrm{mmHg}$, and (or) previously diagnosed hypertension; hypertriglyceridemia $(\mathrm{TG} \geq 1.70 \mathrm{mmol} / \mathrm{L}$ ); low HDL-c $($ HDL-c $<1.04 \mathrm{mmol} / \mathrm{L}){ }^{17}$

\section{Statistical Analysis}

The data were analyzed using SPSS 20.0 version. Continuous variables are shown as mean \pm standard deviation (ones with a normal distribution) and median (interquartile range; ones with a skewed distribution). Categorical variables are expressed as number (percentage). Normal, skewed, and categorical variables were compared using Student's $t$-test, Wilcoxon rank-sum test, and chisquare test, respectively. Logistic regression analysis was conducted to analyze the association between MAU and NC after adjusting for age, lipid profiles, CRP, eGFR, lipidlowering therapy, NC cutoff, glycemic indices, diabetes, antidiabetic therapy, blood pressure, hypertension, antihypertensive therapy, metabolic syndrome and BMI or WC. All reported $P$-values were two-tailed, and a $P$-value $<0.05$ was considered statistically significant.

\section{Results}

\section{Clinical Characteristics of the Study Participants}

The total population had a median age of $61(57-65)$ years (ranging from 40 to 80 years), a median NC of 35.0 $(32.8-37.7) \mathrm{cm}$ [38.0 (36.0-39.8) $\mathrm{cm}$ for men and 33.4 (31.8-34.8) $\mathrm{cm}$ for women], and a median UACR of 7.90 $(5.17-14.55) \mathrm{mg} / \mathrm{g}$. The prevalence of MAU was $11.2 \%$ (210/1882). As listed in Table 1, the values of NC were significantly higher in individuals with MAU in both men and women [38.9 (37.2-40.8) versus $37.9(36.0-39.7) \mathrm{cm}$, $P=0.004$ in men and $34.0(32.3-35.8)$ versus 33.3 $(31.7-34.7) \mathrm{cm}, P=0.001$ in women]. Moreover, participants with MAU had higher values of BMI, WC, SBP, DBP, FPG, 2hPG, $\mathrm{HbA}_{1 \mathrm{c}}$, fasting insulin, HOMA-IR, CRP, and UACR and proportions of hypertension (all $P<0.05)$ than the participants without MAU, and there were no significant differences between two groups in the values of TC, LDL-c, and eGFR (all $P>0.05$ ), irrespective of gender. Furthermore, individuals with MAU had a higher TG $(P<0.001)$ and a lower HDL-c $(P=0.003)$ than those without MAU, in women.

\section{Comparison of MAU in Different NC Groups}

The participants were divided into different groups according to gender and $\mathrm{NC}$ levels. There were 460 individuals with normal $\mathrm{NC}, 356$ individuals with elevated $\mathrm{NC}$ in men and 735 individuals with normal $\mathrm{NC}$, and 331 individuals with elevated $\mathrm{NC}$ in women. As shown in Figure 1, the prevalence of MAU in men was $6.74 \%(31 / 460)$ and $12.36 \%(44 / 356)$ for those with normal and elevated $\mathrm{NC}$, respectively, demonstrating that the prevalence significantly increased in those with elevated NC $(P=0.007)$. Similarly, in women, participants with elevated $\mathrm{NC}$ had higher prevalence of MAU than those with normal NC (18.13\% versus $10.20 \%, P<$ 0.001). Furthermore, UACR of individuals with elevated $\mathrm{NC}$ were also significantly higher than those with normal $\mathrm{NC}$ in both men and women [7.09 (4.49-13.73) versus $6.35(4.45-10.14) \mathrm{mg} / \mathrm{g}, P=0.021$ for men; 10.47 (6.71-21.79) versus $8.62(5.64-15.21) \mathrm{mg} / \mathrm{g}, P<0.001$ for women; Figure 2].

\section{The Association of MAU with NC and Other Measurements}

As shown in Table 2, taking MAU as the dependent variable and NC elevation as the independent variable for logistic regression analysis, there were significant and positive associations between elevated $\mathrm{NC}$ and the increased risk of MAU in both men and women [odds ratio $(\mathrm{OR})=1.952(1.205-3.161), P=0.007$ for men and $\mathrm{OR}=1.875(1.291-2.725), P=0.001$ for women] after adjusting for age, TG, HDL-c, LDL-c, CRP, eGFR and lipid-lowering therapy in model 1 . In model 2 , although further adjustments were made for glycemic indices on the basis of model 1, elevated $\mathrm{NC}$ remained positively correlated with the increased risk of MAU $(P=$ 0.007 for men and 0.009 for women). If we further adjusted for blood pressure related indices in model 3 and metabolic syndrome in model 4 , the results showed that elevated $\mathrm{NC}$ brought about an additional $69.3 \%$ risk of MAU in men [OR $=1.693(1.031-2.781), P=0.037$ ] and the positive correlation between elevated $\mathrm{NC}$ and risk of MAU in women disappeared $(P=0.131)$. Further adjusting for BMI based on model 4 did not change the 
Table I Characteristics of the Study Participants

\begin{tabular}{|c|c|c|c|c|c|c|}
\hline \multirow[t]{3}{*}{ Variable } & \multicolumn{2}{|c|}{ Men } & & \multicolumn{2}{|c|}{ Women } & \multirow{3}{*}{$P$} \\
\hline & \multicolumn{2}{|c|}{ MAU } & \multirow[t]{2}{*}{$P$} & \multicolumn{2}{|c|}{ MAU } & \\
\hline & No $(n=74 I)$ & Yes $(n=75)$ & & No $(n=931)$ & Yes $(n=135)$ & \\
\hline Age (years) & $62(56-66)$ & $62(56-66)$ & 0.844 & $61(57-65)$ & $62(58-65)$ & 0.011 \\
\hline Height (cm) & $170.0(166.0-173.0)$ & $169.0(165.0-172.0)$ & 0.436 & $158.0(154.5-161.0)$ & $158.0(155.0-163.0)$ & 0.142 \\
\hline Weight (kg) & $70.1(64.2-76.7)$ & $73.0(67.3-79.5)$ & 0.008 & $58.3(53.4-64.5)$ & $62.6(56.3-68.6)$ & $<0.001$ \\
\hline BMI $\left(\mathrm{kg} / \mathrm{m}^{2}\right)$ & $24.57 \pm 3.02$ & $25.73 \pm 2.84$ & $<0.001$ & $23.76 \pm 3.16$ & $25.07 \pm 3.52$ & $<0.001$ \\
\hline WC (cm) & $87.0(82.0-93.0)$ & $93.0(85.0-96.5)$ & $<0.001$ & $81.0(76.0-87.0)$ & $85.0(78.0-92.0)$ & $<0.001$ \\
\hline $\mathrm{NC}(\mathrm{cm})$ & $37.9(36.0-39.7)$ & $38.9(37.2-40.8)$ & 0.004 & $33.3(31.7-34.7)$ & $34.0(32.3-35.8)$ & 0.001 \\
\hline $\mathrm{SBP}(\mathrm{mmHg})$ & $134(123-146)$ & $144(130-156)$ & $<0.001$ & $128(|17-| 40)$ & $139(127-154)$ & $<0.001$ \\
\hline $\mathrm{DBP}(\mathrm{mmHg})$ & $80(74-87)$ & $83(77-92)$ & 0.004 & $75(69-82)$ & $78(72-85)$ & 0.001 \\
\hline FPG (mmol/L) & $5.85(5.45-6.53)$ & $6.07(5.49-7.28)$ & 0.033 & $5.78(5.42-6.32)$ & 6.01 (5.54-7.09) & $<0.001$ \\
\hline $2 \mathrm{hPG}(\mathrm{mmol} / \mathrm{L})$ & $7.72(6.07-9.73)$ & $8.61(6.93-13.15)$ & 0.008 & $7.50(6.14-9.78)$ & $8.46(6.65-11.86)$ & $<0.001$ \\
\hline $\mathrm{HbA} \mathrm{Ic}_{\mathrm{Ic}}(\%)$ & $5.7(5.4-6.1)$ & $5.9(5.6-6.4)$ & $<0.001$ & $5.8(5.5-6.1)$ & $6.1(5.7-6.8)$ & $<0.001$ \\
\hline Fasting insulin (mU/L) & $8.40(5.84-12.18)$ & $10.62(6.63-14.97)$ & 0.006 & 9.38 (6.65-12.99) & $11.08(8.13-15.79)$ & $<0.001$ \\
\hline HOMA-IR & $2.24(1.56-3.45)$ & $2.89(1.76-4.78)$ & 0.001 & $2.45(1.70-3.56)$ & $3.12(2.12-4.59)$ & $<0.001$ \\
\hline $\mathrm{TC}(\mathrm{mmol} / \mathrm{L})$ & $5.06(4.49-5.72)$ & $5.24(4.69-5.69)$ & 0.124 & $5.58(5.01-6.30)$ & $5.61(4.96-6.21)$ & 0.420 \\
\hline TG $(\mathrm{mmol} / \mathrm{L})$ & $1.46(1.04-2.21)$ & $1.62(1.12-2.80)$ & 0.135 & $1.38(0.97-1.94)$ & $1.56(1.21-2.24)$ & $<0.001$ \\
\hline $\mathrm{HDL}-\mathrm{c}(\mathrm{mmol} / \mathrm{L})$ & $1.23(1.08-1.45)$ & $1.16(0.95-1.39)$ & 0.078 & $1.50(1.29-1.76)$ & $1.42(1.19-1.65)$ & 0.003 \\
\hline LDL-c (mmol/L) & $3.13 \pm 0.80$ & $3.30 \pm 0.71$ & 0.084 & $3.42 \pm 0.85$ & $3.41 \pm 0.78$ & 0.912 \\
\hline CRP (mg/L) & $0.82(0.38-1.64)$ & $1.11(0.67-2.10)$ & 0.009 & $1.00(0.50-1.76)$ & $1.27(0.67-3.12)$ & 0.002 \\
\hline eGFR $\left(\mathrm{mL} / \mathrm{min} / \mathrm{l} .73 \mathrm{~m}^{2}\right)$ & $93.89(86.98-100.26)$ & $95.31(84.26-104.11)$ & 0.592 & $97.19(91.65-102.12)$ & $98.68(92.36-103.96)$ & 0.200 \\
\hline UACR (mg/g) & $6.04(4.31-9.77)$ & $60.65(41.11-126.11)$ & $<0.001$ & $8.05(5.60-12.45)$ & $51.25(36.39-98.35)$ & $<0.001$ \\
\hline Hypertension, n (\%) & $415(56.0)$ & $56(74.7)$ & 0.002 & $400(43.0)$ & $95(70.4)$ & $<0.001$ \\
\hline Diabetes, n (\%) & $198(26.7)$ & $28(37.3)$ & 0.058 & $211(22.7)$ & $59(43.7)$ & $<0.001$ \\
\hline Metabolic syndrome, n (\%) & $327(44.1)$ & $45(60.0)$ & 0.010 & $292(31.4)$ & $69(51.1)$ & $<0.001$ \\
\hline Lipid-lowering therapy, n (\%) & $40(5.4)$ & $2(2.7)$ & 0.418 & $76(8.2)$ & $13(9.6)$ & 0.617 \\
\hline Antidiabetic therapy, n (\%) & $74(10.0)$ & II (14.7) & 0.231 & $70(7.5)$ & $24(17.8)$ & $<0.001$ \\
\hline Antihypertensive therapy, n (\%) & $201(27.1)$ & $28(37.3)$ & 0.079 & $226(24.3)$ & $60(44.4)$ & $<0.001$ \\
\hline
\end{tabular}

Note: Data were expressed as mean \pm standard deviation for normally distributed variables or the median (interquartile range) for skewed-distribution variables.

Abbreviations: MAU, microalbuminuria; BMI, body mass index; WC, waist circumference; NC, neck circumference; SBP, systolic blood pressure; DBP, diastolic blood pressure; FPG, fasting plasma glucose; 2hPG, 2-h plasma glucose; $\mathrm{HbA}_{\mathrm{Ic}}$, glycated hemoglobin $\mathrm{A}_{\mathrm{Ic}}$; HOMA-IR, homeostasis model assessment-insulin resistance index; TC, total cholesterol; TG, triglyceride; HDL-c, high-density lipoprotein cholesterol; LDL-c, low-density lipoprotein cholesterol; CRP, C-reactive protein; eGFR, estimated glomerular filtration rate; UACR, urine albumin-to-creatinine ratio.

association between elevated $\mathrm{NC}$ and risk of MAU in men $(P=0.037)$. However, the positive association in men disappeared after further adjusting for $\mathrm{WC}$ on the basis of model $4(P=0.449)$

\section{Discussion}

This study found that the average UACR value and prevalence of MAU in participants with elevated $\mathrm{NC}$ were significantly increased in the community population. There 


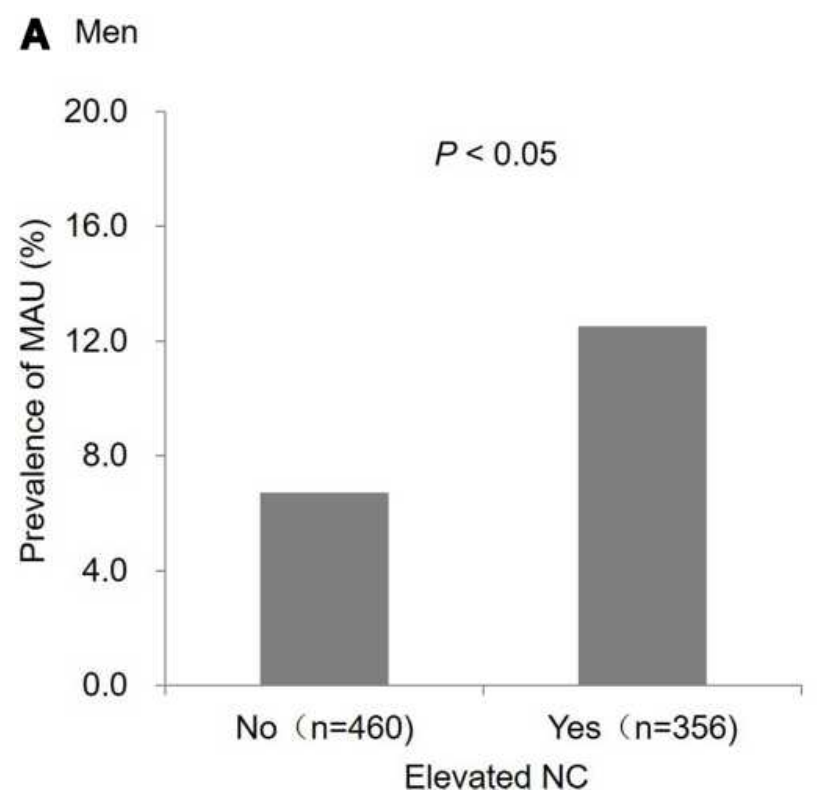

B Women

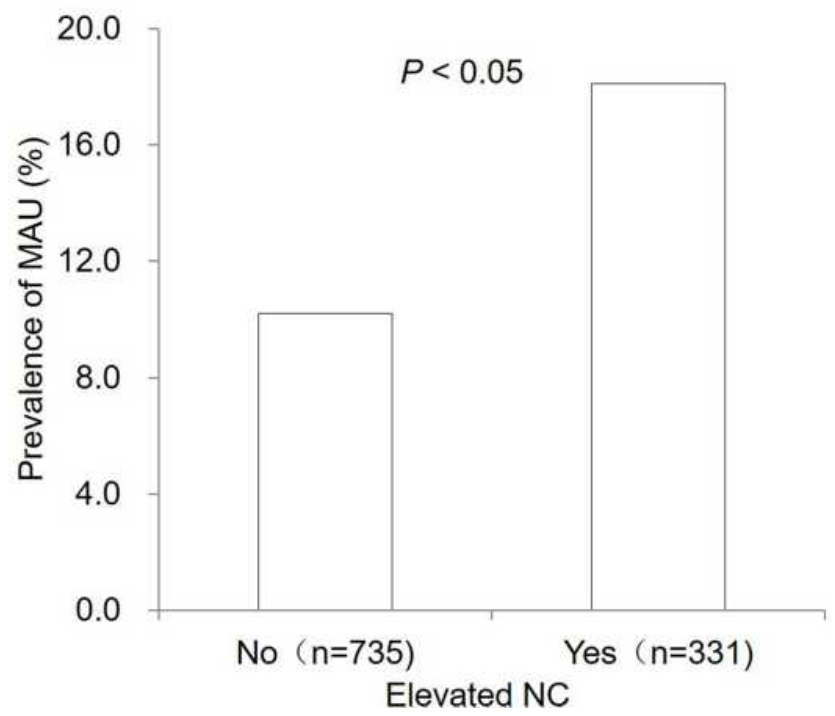

Figure I Prevalence of MAU according to elevated NC groups in men (A) and women (B).

Abbreviations: MAU, microalbuminuria; NC, neck circumference.

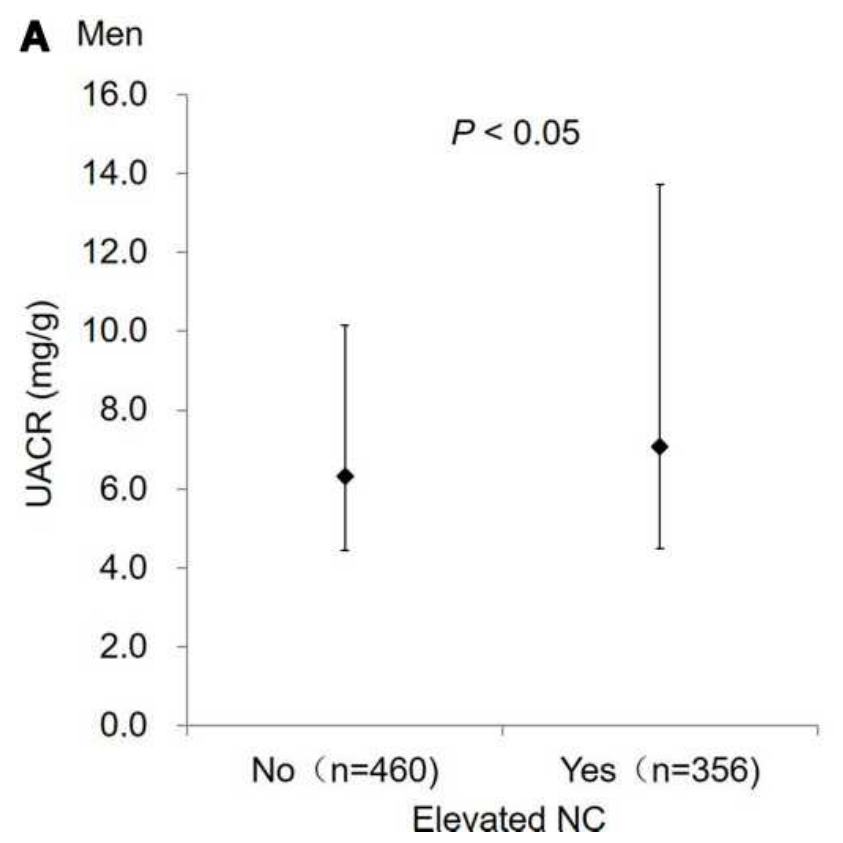

B Women

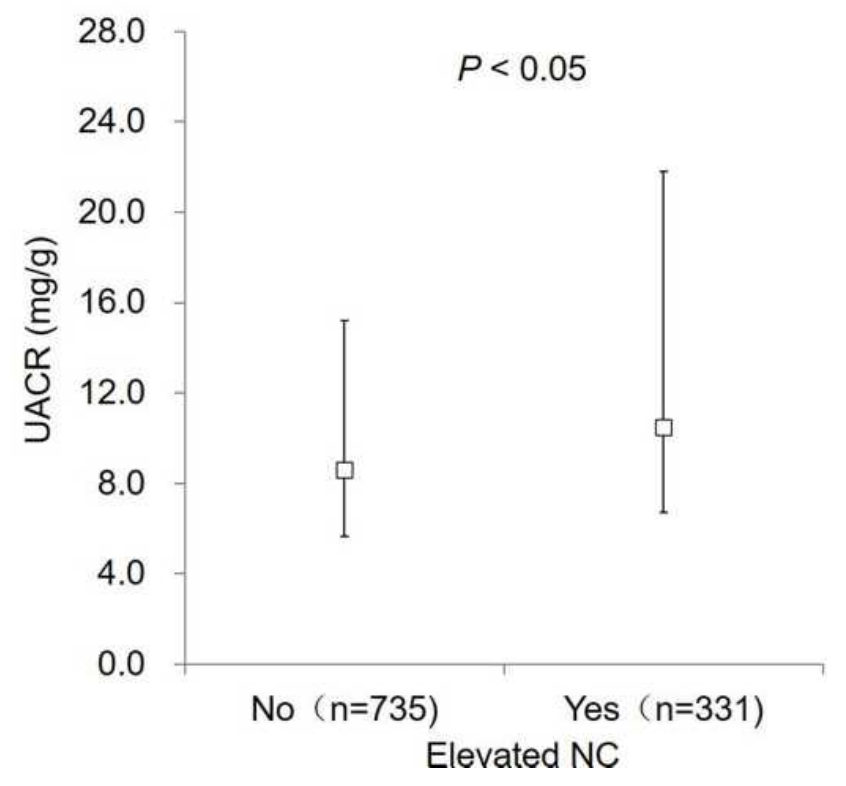

Figure 2 Distribution of UACR according to elevated NC groups in men (A) and women (B).

Abbreviations: UACR, urine albumin-to-creatinine ratio; NC, neck circumference.

was a positive correlation between elevated $\mathrm{NC}$ and the risk of MAU in men, independent of confounding factors, including renal function, lipid profiles, glycemic indices, and blood pressure. However, elevated NC did not contribute to MAU risk independent of metabolic factors in women.
Albuminuria is one of the earliest markers of glomerular diseases and an important indicator of poor renal prognosis. The guideline updated by The Kidney Disease: Improving Global Outcomes (KDIGO) organization in 2013 summarized the relationship between increased proteinuria and a variety of important outcomes, 
Table 2 Logistic Regression Analysis Showing the Variables Associated with MAU

\begin{tabular}{|c|c|c|c|c|}
\hline \multirow[t]{2}{*}{ Variables } & \multicolumn{2}{|l|}{ Men } & \multicolumn{2}{|l|}{ Women } \\
\hline & OR $(95 \% \mathrm{Cl})$ & $P$ & OR $(95 \% \mathrm{Cl})$ & $\mathbf{P}$ \\
\hline \multicolumn{5}{|l|}{ Multivariate Model I } \\
\hline Age & - & - & $1.079(1.037-1.124)$ & $<0.001$ \\
\hline eGFR & - & - & $1.036(1.012-1.061)$ & 0.004 \\
\hline CRP & - & - & $1.042(1.002-1.084)$ & 0.042 \\
\hline NC cutoff & $1.952(1.205-3.161)$ & 0.007 & $1.875(1.291-2.725)$ & 0.001 \\
\hline \multicolumn{5}{|l|}{ Multivariate Model 2} \\
\hline $\mathrm{HbA}_{\mathrm{Ic}}$ & $1.340(1.119-1.605)$ & 0.001 & $1.612(1.356-1.917)$ & $<0.001$ \\
\hline NC cutoff & $1.954(1.201-3.178)$ & 0.007 & $1.658(1.132-2.428)$ & 0.009 \\
\hline \multicolumn{5}{|l|}{ Multivariate Model 3} \\
\hline $\mathrm{HbA}_{\mathrm{Ic}}$ & $1.320(1.095-1.590)$ & 0.004 & $1.512(1.265-1.806)$ & $<0.001$ \\
\hline SBP & $1.022(1.009-1.036)$ & 0.001 & $1.031(1.020-1.043)$ & $<0.001$ \\
\hline NC cutoff & $\mathrm{I} .693(\mathrm{I} .03 \mathrm{I}-2.78 \mathrm{I})$ & 0.037 & - & - \\
\hline Antihypertensive therapy & - & - & $1.587(1.058-2.380)$ & 0.025 \\
\hline \multicolumn{5}{|l|}{ Multivariate Model 4} \\
\hline $\mathrm{HbA}_{\mathrm{Ic}}$ & $1.320(1.095-1.590)$ & 0.004 & $1.512(1.265-1.806)$ & $<0.001$ \\
\hline SBP & $1.022(1.009-1.036)$ & 0.001 & $1.031(1.020-1.043)$ & $<0.001$ \\
\hline NC cutoff & $\mathrm{I} .693(\mathrm{I} .03 \mathrm{I}-2.78 \mathrm{I})$ & 0.037 & - & - \\
\hline Antihypertensive therapy & - & - & $1.587(1.058-2.380)$ & 0.025 \\
\hline
\end{tabular}

Notes: Model I included age, TG, HDL-c, LDL-c, CRP, eGFR, lipid-lowering therapy and NC cutoff. Model 2 included age, TG, HDL-c, LDL-c, CRP, eGFR, lipid-lowering therapy, NC cutoff, $\mathrm{HbA}_{\mathrm{Ic}}$, HOMA-IR, diabetes and antidiabetic therapy. Model 3 included age, TG, HDL-c, LDL-c, CRP, eGFR, lipid-lowering therapy, NC cutoff, HbA, HOMA-IR, diabetes, antidiabetic therapy, SBP, DBP, hypertension and antihypertensive therapy. Model 4 included age, TG, HDL-c, LDL-c, CRP, eGFR, lipid-lowering therapy, $\mathrm{NC}$ cutoff, $\mathrm{HbA}_{\mathrm{Ic}}$, HOMA-IR, diabetes, antidiabetic therapy, SBP, DBP, hypertension, antihypertensive therapy and metabolic syndrome.

Abbreviations: MAU, microalbuminuria; eGFR, estimated glomerular filtration rate; CRP, C-reactive protein; NC, neck circumference; HbA ${ }_{I c}$, glycated hemoglobin $\mathrm{A}_{I c}$; SBP, systolic blood pressure; TG, triglyceride; HDL-c, high-density lipoprotein cholesterol; LDL-c, low-density lipoprotein cholesterol; HOMA-IR, homeostasis model assessment-insulin resistance index; DBP, diastolic blood pressure.

including all-cause mortality, cardiovascular disease, kidney failure, and acute kidney injury for the first time, revealed that MAU (UACR 30-299 mg/g) had indispensable clinical significance in both diabetic and nondiabetic participants, and assigned great importance to the laboratory measurement of UACR. ${ }^{18,19}$

Recently, some studies have explored the relationships between traditional anthropometric indicators and MAU. $^{20-24}$ A cross-sectional study involving 8600 community residents over the age of 40 in China investigated the relationships between $\mathrm{BMI}, \mathrm{WC}$, body fat content, and UACR. The results showed that after adjusting for age, gender, physical activity, FPG, TG, LDL-c, $\mathrm{HbA}_{1 \mathrm{c}}$, HOMA-IR, and SBP, taking the lowest quartile of BMI and $\mathrm{WC}$ as reference, the risk of MAU in the highest quartile of BMI and WC increased by $65 \%$ and $73 \%$, respectively. ${ }^{20}$ Another Chinese study conducted in 341 newly diagnosed type 2 diabetes patients demonstrated that the prevalence of MAU increased with an increase in visceral fat index. The risk of MAU increased by $94 \%$ per one standard deviation increase in the visceral fat index, revealing the predictive ability of visceral fat index in MAU. ${ }^{21}$ A study based on the Korean National Health and Nutrition Examination Survey 2011-2014 data (19,331 participants, $\geq 19$ years old) found that compared to individuals with normal BMI, overweight/obese individuals possessed an additional risk of approximately 50\% of increased UACR value. ${ }^{22}$ A longitudinal populationbased cohort involving 26,960 participants in the REGARDS study demonstrated that there was no significant association between elevated BMI and the incidence of end-stage renal disease. Those with elevated WC had a 3.97-fold increased risk of end-stage renal disease. However, after adjusting for obesity-related comorbidities and baseline values of UACR and eGFR, this correlation disappeared. $^{23}$ The results from the study conducted by Dittmann et al showed that there was a U-shaped association between WC and MAU in the German general population, suggesting that both obese and very thin people had an increased risk of renal insufficiency. ${ }^{24}$ Although the results on the relationships between the traditional anthropological indicators such as BMI, WC, and MAU were 
slightly discrepant because of the differences in study population and diagnostic criteria, most of the studies suggested that the systemic or visceral fat accumulation reflected by BMI or WC were positively correlated with the prevalence of MAU.

As an anthropological indicator of obesity proposed in recent years, $\mathrm{NC}$ was revealed to be associated with MAU in few studies. ${ }^{25-27}$ A small sample study in South Africa comprising 101 black African men and 99 women showed that after adjusting for confounding factors, a significant and positive correlation between $\mathrm{NC}$ and MAU was observed only in women $(\beta=0.52, P<0.01){ }^{25}$ Another study from Taiwan in 177 consecutive patients who visited the outpatient department of cardiology found that after adjusting for age and creatinine, there was no significant correlation between $\mathrm{NC}$ and $\mathrm{MAU}$ in men and $\mathrm{NC}$ was significantly and positively correlated with MAU in women, with higher linear regression correlation coefficient than BMI and WC. ${ }^{26}$ The results of the abovementioned studies are inconsistent as a result of differences in the study population and design; moreover, there is a lack of evidence from the Chinese population. In this study, we found that elevated $\mathrm{NC}$ was independently and positively correlated with the risk of MAU after adjusting for age, lipid profile, and glycemic indices. The correlation disappeared in women and remained in men after further adjusting for blood pressure. In other words, elevated NC was an independent determinant for MAU in men, irrespective of lipid profile, glycemic indices, and blood pressure.

Owing to the difference in study population size, the associations between $\mathrm{NC}$ and MAU in men and women are inconsistent. In this study, we only observed the independent association between elevated $\mathrm{NC}$ and MAU in men after adjusting for blood pressure, lipid profile, glycemic indices, CRP, and other metabolic factors. The difference in the absolute content of upper-body subcutaneous fat between men and women may account for the results in our study. Upper-body subcutaneous fat has been considered to be the main reservoir of circulating non-esterified fatty acids (NEFAs). NEFAs affected the production of vascular nitric oxide, enhanced endothelial dysfunction, and impaired vascular reactivity. In addition, the renal uptake of NEFAs in the proximal tubules also caused tubulointerstitial inflammation and fibrosis. ${ }^{28,29}$

There are some limitations in the current study. Given the single-center study design, the conclusions of this study need to be verified in a multi-center, large-cohort study. Another limitation is the fact that it is a crosssectional study; therefore, the predictive efficiency of $\mathrm{NC}$ on the development of MAU needs to be verified using longitudinal studies in the future. In addition, the study population had higher prevalence of diabetes and hypertension than the general population and further studies in a general population are needed to confirm the findings.

\section{Conclusion}

In the Chinese community population, there was an independent and positive association between elevated $\mathrm{NC}$ and the risk of MAU only in men.

\section{Data Sharing Statement}

The data that support the findings of this study are available on request from the corresponding author (Yuqian Bao, yqbao@sjtu.edu.cn). The data are not publicly available due to privacy or ethical restrictions.

\section{Ethical Approval and Informed Consent}

The study was approved by the Ethics Committee of Shanghai Jiao Tong University Affiliated Sixth People's Hospital, and all procedures were performed in accordance with the 1964 Declaration of Helsinki and its later amendments or comparable ethical standards. The study methods and potential risks were fully explained to all participants, and each participant provided a written informed consent prior to enrollment.

\section{Funding}

This work was supported by the Shanghai Municipal Science and Technology Commission Medical Guide Project (19411964300).

\section{Disclosure}

The authors declare no potential conflicts of interest.

\section{References}

1. Luo Y, Ma X, Shen Y, et al. Neck circumference as an effective measure for identifying cardio-metabolic syndrome: a comparison with waist circumference. Endocrine. 2017;55(3):822-830. doi:10.1007/s12020-016-1151-y

2. Yang GR, Yuan SY, Fu HJ, et al. Neck circumference positively related with central obesity, overweight, and metabolic syndrome in Chinese subjects with type 2 diabetes: beijing Community Diabetes Study 4. Diabetes Care. 2010;33(11):2465-2467. doi:10.2337/dc10-0798

3. Xu Y, Jian C, Ma X, Shen Y, Wang Y, Bao Y. Comparison of neck and waist circumferences for identifying subclinical atherosclerosis in a community-based population. Diabetes Metab Res Rev. 2020;e3382.

4. Jian C, Xu Y, Ma X, Shen Y, Wang Y, Bao Y. Neck circumference is an effective supplement for nonalcoholic fatty liver disease screening in a community-based population. Int $J$ Endocrinol. 2020;2020:7982107. doi:10.1155/2020/7982107 
5. Arias-Tellez MJ, Acosta FM, Garcia-Rivero Y, et al. Neck adipose tissue accumulation is associated with higher overall and central adiposity, a higher cardiometabolic risk, and a pro-inflammatory profile in young adults. Int J Obes (Lond). 2020;2:1-3. doi:10.1038/ s41366-020-00701-5

6. Preis SR, Pencina MJ, D’Agostino RB Sr, Meigs JB, Vasan RS, Fox CS. Neck circumference and the development of cardiovascular disease risk factors in the Framingham Heart Study. Diabetes Care. 2013;36(1):e3. doi:10.2337/dc12-0738

7. Abdelhafiz AH, Ahmed S, El Nahas M. Microalbuminuria: marker or maker of cardiovascular disease. Nephron Exp Nephrol. 2011;119 (Suppl 1):e6-10. doi:10.1159/000328015

8. Singh A, Satchell SC. Microalbuminuria: causes and implications. Pediatr Nephrol. 2011;26(11):1957-1965. doi:10.1007/s00467-0111777-1

9. De Jong PE, Brenner BM. From secondary to primary prevention of progressive renal disease: the case for screening for albuminuria. Kidney Int. 2004;66(6):2109-2118. doi:10.1111/j.15231755.2004.66001.x

10. Hillege HL, Fidler V, Diercks GF, et al. Urinary albumin excretion predicts cardiovascular and noncardiovascular mortality in general population. Circulation. 2002;106(14):1777-1782. doi:10.1161/01. CIR.0000031732.78052.81

11. Adachi H. Microalbuminuria is an independent prognostic information for cardiovascular disease. Atherosclerosis. 2014;237 (1):106-107. doi:10.1016/j.atherosclerosis.2014.05.964

12. Xue J, Li B, Wang J, et al. Association between neck circumference and the risk of decreased estimated glomerular filtration rate in the general population of China: a cross-sectional study. BioMed Res Int. 2020;2020:3496328. doi:10.1155/2020/3496328

13. Levey AS, Stevens LA, Schmid $\mathrm{CH}$, et al. A new equation to estimate glomerular filtration rate. Ann Intern Med. 2009;150 (9):604-612. doi:10.7326/0003-4819-150-9-200905050-00006

14. American Diabetes Association. Standards of medical care in diabetes-2010. Diabetes Care. 2010;33(Suppl 1):S11-61. doi:10.2337/ de10-S011

15. Unger T, Borghi C, Charchar F, et al. 2020 International Society of Hypertension global hypertension practice guidelines. J Hypertens. 2020;38(6):982-1004. doi:10.1097/HJH.0000000000002453

16. Khosla N, Sarafidis PA, Bakris GL. Microalbuminuria. Clin Lab Med. 2006;26(3):635-53, vi-vii. doi:10.1016/j.cll.2006.06.005

17. Chinese Diabetes Society. Guideline for the prevention and treatment of type 2 diabetes mellitus in China (2020 edition). Chin J Diabetes Mellitus. 2021;13(4):315-409.

18. Stevens PE, Levin A. Evaluation and management of chronic kidney disease: synopsis of the kidney disease: improving global outcomes 2012 clinical practice guideline. Ann Intern Med. 2013;158 (11):825-830. doi:10.7326/0003-4819-158-11-201306040-00007
19. Lamb EJ, Levey AS, Stevens PE. The Kidney Disease Improving Global Outcomes (KDIGO) guideline update for chronic kidney disease: evolution not revolution. Clin Chem. 2013;59(3):462-465. doi:10.1373/clinchem.2012.184259

20. Ren M, Sun K, Li F, et al. Association between obesity measures and albuminuria: a population-based study. J Diabetes Complications. 2016;30(3):451-456. doi:10.1016/j.jdiacomp.2015.12.007

21. Wen J, Yuan H. Independent association between the visceral adiposity index and microalbuminuria in patients with newly diagnosed type 2 diabetes. Diabetes Metab Res Rev. 2020;36(1):e3198. doi: $10.1002 /$ dmrr.3198

22. Kim YJ, Hwang SD, Oh TJ, et al. Association between obesity and chronic kidney disease, defined by both glomerular filtration rate and albuminuria, in Korean adults. Metab Syndr Relat Disord. 2017;15 (8):416-422. doi:10.1089/met.2017.0053

23. Kramer H, Gutiérrez OM, Judd SE, et al. Waist circumference, body mass index, and ESRD in the REGARDS (Reasons for Geographic and Racial Differences in Stroke) study. Am J Kidney Dis. 2016;67 (1):62-69. doi:10.1053/j.ajkd.2015.05.023

24. Dittmann K, Hannemann A, Wallaschofski H, et al. U-shaped association between central body fat and the urinary albumin-tocreatinine ratio and microalbuminuria. BMC Nephrol. 2013;14 (1):87. doi:10.1186/1471-2369-14-87

25. Hoebel S, de Ridder JH, Malan L. The association between anthropometric parameters, the metabolic syndrome and microalbuminuria in black Africans: the SABPA study. Cardiovasc J Afr. 2010;21 (3):148-152. doi:10.5830/CVJA-2010-025

26. Liu YF, Chang ST, Lin WS, et al. Neck circumference as a predictive indicator of $\mathrm{CKD}$ for high cardiovascular risk patients. BioMed Res Int. 2015;2015:745410.

27. Yoon CY, Park JT, Jhee JH, et al. Neck circumference predicts renal function decline in overweight women: a community-based prospective cohort study. Medicine. 2016;95(36):e4844. doi:10.1097/ MD.0000000000004844

28. Kamijo A, Kimura K, Sugaya T, et al. Urinary free fatty acids bound to albumin aggravate tubulointerstitial damage. Kidney Int. 2002;62 (5):1628-1637. doi:10.1046/j.1523-1755.2002.00618.x

29. Jensen MD, Haymond MW, Rizza RA, et al. Influence of body fat distribution on free fatty acid metabolism in obesity. $J$ Clin Invest. 1989;83(4):1168-1173. doi:10.1172/JCI113997

Diabetes, Metabolic Syndrome and Obesity: Targets and Therapy

\section{Publish your work in this journal}

Diabetes, Metabolic Syndrome and Obesity: Targets and Therapy is an international, peer-reviewed open-access journal committed to the rapid publication of the latest laboratory and clinical findings in the fields of diabetes, metabolic syndrome and obesity research. Original research, review, case reports, hypothesis formation, expert opinion and commentaries are all considered for publication. The manuscript management system is completely online and includes a very quick and fair peer-review system, which is all easy to use. Visit http://www.dovepress.com/testimonials.php to read real quotes from published authors. 